\title{
A inserção da mentoria na matriz curricular de um curso de Medicina: relato de experiência
}

The inclusion of mentoring in the medical course curriculum: an experience report

\author{
Maria Helena Senger ${ }^{1}$ (D) mhsenger@pucsp.br \\ Luiz Ferraz de Sampaio Neto' (D) Ifsampaio@pucsp.br \\ Marta Wey Vieira' (1) mwvieira@pucsp.br \\ Godofredo Campos Borges' 1 (1) gcborges@pucsp.br \\ Juliana Abeche Fermozelli' (1) jafermozelli@pucsp.br \\ Sandro Blasi Esposito' (1) sbesposito@pucsp.br \\ Felipe de Oliveira Teixeira' ${ }^{(1)}$ foteixeira@pucsp.br \\ Fernando Antonio de Almeida' (1) faalmeida@pucsp.br
}

\begin{abstract}
RESUMO
Introdução: Em 2018, foi implantado no curso de Medicina da Faculdade de Ciências Médicas e da Saúde da Pontifícia Universidade Católica de São Paulo um novo projeto pedagógico do curso (PPC). Nessa construção, surgiu a oportunidade de incluir um módulo para a prática da mentoria, pois havia várias questões relacionadas à vida no câmpus que vinham preocupando a comunidade, tais como a necessidade de os alunos e professores apropriarem-se do PPC, o fato de os discentes ainda muito jovens serem afastados da família, estudantes oriundos de escolas tradicionais pouco afeitas às metodologias ativas de ensino-aprendizagem que constituem a base do curso, a avaliação somativa e a recuperação do desempenho insatisfatório, o trote, o convívio com a diversidade, a autonomia do aluno e o processo de formação e identidade do profissional médico.

Relato de experiência: No novo PPC, o módulo de Estudos Orientados garantiu o espaço para a mentoria dentro da grade curricular do primeiro ao terceiro ano, em pequenos grupos protegidos. Ao fim do primeiro ano, a avaliação regular da docência revelou dificuldades que foram superadas com a capacitação dos docentes para a atividade da mentoria, viabilizada com o auxílio de profissionais externos e experientes que ajudaram a dar identidade à mentoria, ajustada às características do curso e do PPC. A avaliação seguinte revelou melhora evidente da percepção dos alunos e professores. Nesse momento, a consulta feita com a maioria dos envolvidos trouxe novas informações que permitiram estabelecer diretrizes norteadoras para a atividade em cada ano do curso.
\end{abstract}

Discussão: Todo o processo de implantação do módulo, dividido em três etapas, foi muito rico e exigiu a participação de todos os envolvidos.

Conclusão: Nossa experiência permite inferir que, por suas características complexas e mutáveis, a mentoria deve ser construída em estreita relação com a comunidade e ajustada às necessidades de cada curso e do seu projeto pedagógico.

Palavras-chave: Educação Médica; Mentores; Profissionalismo; Estudantes de Medicina.

\section{ABSTRACT}

Introduction: In 2018, a new pedagogical project (PP) was implemented in the medical course of the Faculty of Medical and Health Sciences of the Pontifical Catholic University of São Paulo. In this framework the opportunity arose to include a module for the practice of mentoring. At that time there were several issues related to life on campus that had been worrying the community, such as the need for students and teachers to take part in the PP, very young students living away from their families, students from traditional schools unfamiliar with the active teaching-learning methodologies that form the basis of the course, summative assessment and recovery from unsatisfactory performance, hazing, living with diversity, student autonomy and the construction process of the identity of the medical professional.

Experience report: In the new PP, the Guided Studies module guaranteed a space for mentoring within the curriculum from the first to the third year, in small, protected groups. At the end of the first year, the regular teachers' assessment revealed difficulties that were overcome through teacher training in mentoring, supported by experienced teachers who helped to give identity to the mentoring, shaped to the characteristics of the course and the PP. The following evaluation revealed an evident improvement in the perception of students and teachers. A survey was conducted at that time among the majority of those involved, bringing to light new information that allowed for mentoring guidelines for each year of the course to be established.

Discussion: The entire three stages implementation process of the module was very rich and required the participation of all those involved.

Conclusion: This experience allows us to infer that, due to its complex and changeable characteristics, mentoring must be built in close relationship with the community and adjusted to the needs of each course and its pedagogical project.

Keywods: Medical Education; Mentors; Professionalism; Medical Students.

1 Pontifícia Universidade Católica de São Paulo, Sorocaba, São Paulo, Brasil.

Editora: Lia Marcia Cruz da Silveira

Recebido em 03/03/21; Aceito em 10/03/21.

Avaliado pelo processo de double blind review. 


\section{INTRODUÇÃO}

Qual é o material necessário para se esculpir um mentor? E o mentorado? E o programa de mentoria? Tais dúvidas permeavam o grupo gestor do curso de Medicina da Pontifícia Universidade Católica de São Paulo (PUC-SP), câmpus de Sorocaba, em 2018 e se repetiam sistematicamente a cada pessoa consultada para atuar no módulo em que a mentoria é praticada.

$\mathrm{O}$ atual projeto pedagógico do curso (PPC) vem de uma trajetória de profundas modificações desde 2006, com a opção por uma

[...] concepção dialógica, crítico-reflexiva, centrado nas pessoas (estudantes e cidadãos, a quem se direciona o trabalho médico, de modo individual ou coletivo), orientado por objetivos de aprendizagem e/ou competências. Utiliza-se das teorias do construtivismo, da aprendizagem significativa, da pedagogia crítica e é baseado em objetivos educacionais. Por sua flexibilidade e permanente ciclo de avaliação e tomada de decisão, o currículo pode transitar por várias metodologias, desde que guardem a referência da concepção do curso ${ }^{1}$. (p.21)

A necessidade de um acompanhamento dos alunos diferente daquele praticado na tutoria (utilizada na aprendizagem baseada em problemas) ou na preceptoria (praticada nos anos do internato) se apresentava como importante e cada vez mais indispensável. As estratégias da tutoria e da preceptoria, já adotadas, não abordavam, de forma sistemática, temas relevantes para os jovens alunos ingressantes, provenientes de outras cidades e que, em sua maioria, enfrentavam o afastamento de suas famílias pela primeira vez, após um vestibular bastante concorrido. Além disso, o PPC adota metodologias ativas no processo de ensino-aprendizagem que exigem a responsabilidade do aluno pela própria formação, em constante exercício, algo pouco praticado na vida escolar pregressa da maioria dos estudantes. Da mesma forma, a avaliação do desempenho do discente, critério-referenciada e culminando com conceitos (exemplar, satisfatório e insatisfatório), costuma trazer desconforto aos alunos. Todo esse conteúdo do PPC necessita ser apropriado pelos discentes, com suas adequadas justificativas de utilização. Assim, consideramos que um ambiente propício para trocas, discussões de problemas e busca pela superação de dificuldades seria oportuno. Seguindo as definições de Botti et al. ${ }^{2}$, pareceu-nos que os encontros entre mentor e mentorado seriam a melhor estratégia para contemplar tais necessidades, com a expectativa de que poderiam avançar para o compartilhamento de outros temas relevantes para o grupo, ao longo dos anos pré-internato.

É nesse sentido que o presente relato objetiva apresentar a nossa trajetória de três anos de construção do módulo em que se pratica a mentoria e organizá-la em seus principais atributos.

\section{RELATO DE EXPERIÊNCIA}

Em 2018, foi implantado no curso de Medicina da Faculdade de Ciências Médicas e da Saúde (FCMS) da PUC-SP um novo PPC, e, nessa construção, surgiu a possibilidade de incluir um módulo para a prática da mentoria. Essa intenção era acalentada por vários professores, alunos e gestores do curso, mas a situação nunca se mostrava apropriada para o formato pretendido, ou seja, ter horário protegido na matriz curricular semanal (duas horas), desenvolver-se em pequenos grupos (dez alunos por mentor) e ser mantido nos três anos pré-internato. Também em 2018 iniciou-se o internato a partir do quarto ano do curso, modificação já prevista na reforma curricular anterior.

O exercício da mentoria foi concebido como o local apropriado para a discussão de questões que sempre preocuparam os gestores e a comunidade acadêmica, tais como a apropriação do PPC por toda a comunidade, o "trote", a vida universitária, a profissão médica, a aprendizagem reflexiva, os processos de ensino-aprendizagem, de avaliação e de recuperação de desempenhos insatisfatórios, além da autonomia do aluno.

\section{Necessidade e oportunidade}

Diante da oportunidade que se apresentava, do desenho modular do curso e das características pretendidas, o módulo no qual se pratica a mentoria foi denominado Estudos Orientados e viabilizado após aprovação pelos órgãos de gestão acadêmica.

A ementa e os objetivos do módulo estão apresentados no Quadro 1.

Quadro 1. Ementa e objetivos do módulo Estudos Orientados.

Ementa:Reflexãono processo deaprendizagem. Autoavaliação. Identidade profissional. Desenvolvimento interpessoal, psicossocial, educacional e profissional. Autonomia.

\section{Objetivos:}

- Promover o acompanhamento sistemático dos alunos, de acordo com os eixos e módulos em desenvolvimento no respectivo ano letivo, para, sem paternalismo:

- Fomentar e fortalecer a formação da identidade profissional médica.

- Favorecer a reflexão, o raciocínio crítico e a competência relacional.

- Auxiliar a prevenir e/ou remediar desempenhos acadêmicos considerados insatisfatórios.

- Possibilitar encaminhamentos mais adequados de alunos necessitados de outros níveis e tipos de assistências.

- Criar condições em que o aluno reconheça suas necessidades de aprendizagem e as formas para satisfazê-las com sucesso.

- Co-responsabilizar os discentes e com eles pactuar o entendimento, as formas e os prazos para o alcance de metas de bom desempenho pessoal e acadêmico.

Fonte: PUC-SP ${ }^{1}$ p. 96. 
O desafio que se apresentava agora era conseguir professores com o perfil desejado, descrito no PPC (Quadro 2), para que pudessem atuar como mentores. Foi necessária uma busca ativa de professores para tal exercício.

Enfrentamos o desafio de implantar o módulo no início de 2018 contando apenas com reuniões e encontros locais entre os mentores, e com o suporte da psicóloga que atua no Programa de Atendimento Comunitário, Shelley Arruda Pinhal de Camargo, de grande ajuda nesse processo.

\section{Desenvolvimento docente}

As dificuldades apareceram e foram acertadamente apontadas pelos alunos durante a Avaliação da Docência realizada no segundo semestre de 2018 , em discursos como:

Eu sinceramente ainda não compreendi muito bem o objetivo da mentoria. Eu não me sinto à vontade em desabafar com o meu mentor, pois acho que isso não deveria ser o objetivo da mentoria. Além disso, eu acho que duas horas semanais todas as semanas é muito tempo para pouco assunto. Eu sinceramente sinto que estou perdendo tempo na mentoria.

Mentoria: um espaço que nos é dado, mas que não sabemos bem como usá-lo.

A necessidade de capacitação tornou-se evidente e foi voz corrente entre mentores e alunos. Iniciamos esse processo de capacitação e tivemos três edições (dezembro de 2018, junho de 2019 e fevereiro de 2020), com 16 horas presenciais em cada edição, com as professoras Denise Herdy Afonso e Lia Marcia da Cruz Silveira, sob o tema "Desenvolvimento Docente

Quadro 2. Perfil desejado do mentor para atuar no módulo Estudos Orientados.

- Médico, preferencialmente com formação na área de Educação em Saúde;

- Disponibilidade para atuar por duas horas semanais junto aos alunos e com possibilidade de continuidade desta atividade até o $3^{\circ}$ ano do curso;

- Habilidades para relacionamento interpessoal efetivo, incluindo a prática de feedback apreciativo;

- Capacidade para o cuidado e auxílio aos alunos, promovendo a autoavaliação e evitando complacência, condescendência e/ou o paternalismo desmedidos;

- Organização para alimentar o prontuário do aluno com o relato das atividades executadas e pareceres sobre a avaliação;

- Respeito à diversidade em todos os seus aspectos, tanto em relação aos alunos, como também entre seus pares;

- Assiduidade em reuniões com os demais mentores e professores com periodicidade a ser estipulada na dependência das necessidades apresentadas, com a finalidade de capacitação, educação continuada e discussão sobre o módulo para sua lapidação.

Fonte: PUC-SP ${ }^{1}$ p.180. para a Prática da Mentoria". Entre os objetivos das capacitações, destacamos: reconhecer as trajetórias e a motivação para participar como mentor; conhecer as definições teóricas e as construções práticas relacionadas ao "ser docente", "ser mentor", "ser preceptor", "ser tutor"; discutir as funções que são atribuídas ao mentor no processo formativo na saúde; delinear o perfil do mentor considerando a educação no século XXI; identificar desafios e potencialidades no exercício da mentoria; valorizar as dimensões do processo de trabalho envolvido na mentoria; identificar, a partir das experiências e dos referenciais teóricos, os desafios no processo de mentoria e construir propostas que qualifiquem a atuação do mentor; identificar ligações entre a mentoria como prática pedagógica e o desenvolvimento das competências socioemocionais; experimentar a relação de parceria a partir de uma conexão e um objetivo comum; construir um roteiro de situação de mentoria; exercitar a dramatização (role play) e a reflexão (debriefing); refletir sobre os desafios na prática da mentoria e sobre os caminhos para implantação dessa estratégia de educação e cuidado; avaliar a experiência na oficina. Em cada edição, docentes e discentes participantes da mentoria eram ouvidos em separado e com garantia de sigilo para a liberdade de manifestação. Foi criado um espaço de acolhimento e de análise conjunta.

Já no segundo semestre de 2019, na Avaliação da Docência, a mudança foi perceptível. Segundo um aluno:

\begin{abstract}
A mentoria evoluiu muito do primeiro para o segundo ano, e continua em evolução. Acho muito interessante e necessário esse acompanhamento com o que está acontecendo no módulo, os professores, tutores e preceptores deveriam ouvir mais os mentores, pois eles que acompanham mais de perto os alunos. Acho que seria legal os mentores terem uma atividade individual com os alunos, possibilitando mais privacidade. $O$ meu mentor é sensacional, ajuda demais, prestativo, se importa com os seus alunos, só tenho elogios para esse professor, essa pessoa sensacional, um exemplo a ser seguido, se todos os professores fossem iguais a ele, jamais haveria problemas na faculdade.
\end{abstract}

As capacitações foram, portanto, exitosas no sentido da construção da identidade do mentor que respeitasse as características do curso, algo ímpar quando se reflete sobre os objetivos do módulo a serem alcançados (Quadro 1).

Com o desenvolvimento do módulo ao mesmo tempo para todos os alunos dos três primeiros anos e mantendo a proporção de dez alunos para um mentor, há a necessidade de termos, no mínimo, 30 mentores atuando simultaneamente. Tivemos algumas desistências (seis ao todo) desde a implantação do módulo, por motivos diversos, entre eles a dificuldade de adaptação a uma atividade acadêmica tão flexível e pouco precisa, até mesmo fluida, quando comparada aos conteúdos das áreas biomédicas. 


\section{Avaliar para avançar}

A turma que iniciou o módulo desde o primeiro ano do curso completou o terceiro ano em 2020. Assim, conforme já constava do PPC (p. 180), a necessidade de sua continuidade durante o internato seria objeto de uma avaliação do processo. Realizamos uma consulta, com perguntas abertas e fechadas e outras com respostas em escala Likert, que foi encaminhada de forma virtual para todos os 31 professores e 305 alunos envolvidos. As respostas foram sigilosas, e não houve identificação das diferentes séries. Obtivemos as respostas de $30(96,8 \%)$ professores e de $158(51,8 \%)$ alunos. As perguntas solicitavam a atribuição de uma pontuação de 0 a 10 para a experiência como mentor ou mentorado (sendo 10 a melhor experiência possível). Também foi inquirido se deveria haver continuidade da mentoria durante os três anos de internato, de forma opcional ou obrigatória, em qual frequência e em qual modelo (igual ao atual ou algum outro). A Tabela 1 apresenta os dados obtidos.

Com respeito às linhas norteadoras, estudantes $\mathrm{e}$ professores deram suas sugestões que foram contempladas em uma proposta representada na Figura 1, na forma de grandes categorias que englobam subcategorias, retiradas das sugestões presentes nas respostas às perguntas abertas, de acordo com o ano do curso. Mesmo assim, mentores e alunos também apontaram que encontros individuais e a flexibilização dos conteúdos abordados nestes são tão importantes quanto a segurança trazida pelas linhas norteadoras.

Em 2020, com a intercorrência da pandemia de coronavirus disease 2019 (Covid-19), foi necessário migrar as
Figura 1. Linhas norteadoras (em negrito) e seus componentes para o desenvolvimento da mentoria do primeiro ao terceiro ano do curso de Medicina, propostas após a consulta feita a professores e alunos participantes da atividade.

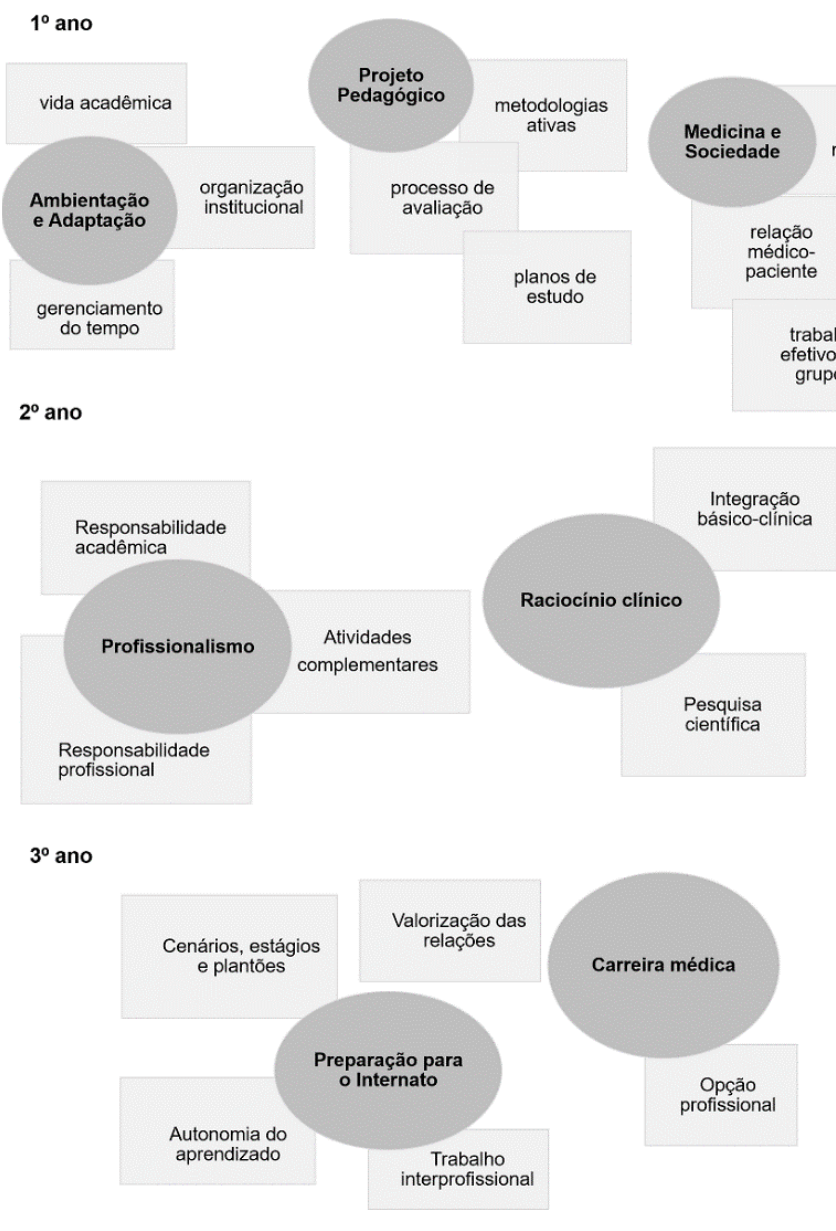

Tabela 1. Frequência das respostas de alunos e professores à consulta sobre a experiência da mentoria (pontuação de 0 a 10 - melhor experiência); necessidade de linhas norteadoras para o primeiro, segundo e terceiro anos; opinião sobre a continuidade da mentoria no Internato e seu modelo.

\begin{tabular}{|c|c|c|c|c|c|c|}
\hline & $\begin{array}{c}\text { Pontuação } \\
\text { da } \\
\text { experiência }\end{array}$ & $\begin{array}{c}\text { Linha } \\
\text { norteadora } \\
\text { do } 1^{\circ} \text { ao } 3^{\circ} \\
\text { ano }\end{array}$ & $\begin{array}{l}\text { Continuidade } \\
\text { no internato }\end{array}$ & Forma & Frequência & Modelo \\
\hline \multirow{4}{*}{$\begin{array}{c}\text { Docentes } \\
n=30 \\
96,8 \%\end{array}$} & & & & \multirow{4}{*}{$\begin{array}{l}\text { Opcional: } \\
79 \%\end{array}$} & Quinzenal: 60\% & Igual ao atual: $37 \%$ \\
\hline & & & & & Mensal: 23\% & Com alunos de diferentes anos: $50 \%$ \\
\hline & $8,9,10: 70 \%$ & NÃO: 20\% & NÃO: 10\% & & Sob demanda: $10 \%$ & Outros: $13 \%$ \\
\hline & $0,9,10.70 \%$ & INAU. $\angle U \%$ & NAU. $10 \%$ & & Outras: $6 \%$ & \\
\hline \multirow{4}{*}{$\begin{array}{c}\text { Alunos } \\
\mathrm{n}=158 \\
51,8 \%\end{array}$} & 1 a $4: 11 \%$ & SIM: $61 \%$ & SIM: 59\% & \multirow{4}{*}{$\begin{array}{l}\text { Opcional: } \\
70 \%\end{array}$} & Quinzenal: $60 \%$ & Igual ao atual: $49 \%$ \\
\hline & $5,6,7: 32 \%$ & & & & Sem resposta: $25 \%$ & Sem resposta: $30 \%$ \\
\hline & $8910.56 \%$ & NÃO: 39\% & NÃO: $41 \%$ & & Mensal: 8\% & Com alunos de diferentes anos: $16 \%$ \\
\hline & $0,0,0.0070$ & & & & Semanal: 7\% & Outros: $4 \%$ \\
\hline
\end{tabular}


atividades de mentoria para o ambiente virtual, de forma síncrona, pela plataforma Microsoft Teams. Foi mais um grande desafio suplantado por professores e alunos. Uma avaliação dessa migração mostrou que $70 \%$ a $80 \%$ dos estudantes consideraram que a mentoria tinha contribuído positivamente para a adaptação às atividades em ambiente virtual e poderia ser mantida de forma remota caso houver necessidade sanitária.

Um incidente durante a migração do ambiente presencial para o virtual foi provocado pela necessidade de gravação, então tida como compulsória para as atividades realizadas remotamente. Os alunos questionaram tal obrigatoriedade, uma vez que lhes foi assegurado que o ambiente da mentoria seria sempre seguro. A reivindicação dos alunos foi discutida entre docentes e gestores, considerada justa e pertinente, e aboliu-se a gravação.

\section{DISCUSSÃO}

Nosso relato de experiência traz a trajetória percorrida pelo curso de Medicina da FCMS-PUC-SP e suas escolhas para a implantação de um modelo de mentoria, aplicado como um módulo longitudinal nos três primeiros anos da graduação. Foi a primeira e única iniciativa desse tipo concretizada no curso que formou sua primeira turma em 1956, mostrando que, mesmo em instituições antigas, as mudanças estratégicas têm espaço, quando embasadas e devidamente apropriadas pela comunidade acadêmica.

Opercurso foianaliticamente divididoem trêsmomentos. No primeiro, em que a oportunidade foi aproveitada em nome da necessidade, criaram-se as condições para a implantação imediata da mentoria. As escolhas não foram fáceis e trouxeram marcas para mentores, mentorados e gestores. A insegurança, a inexperiência, os conflitos, as cobranças e a sensação de ineficiência apareceram como consequência das experiências vivenciadas pela nova atividade acadêmica. Porém, com a oportunidade criada, os riscos foram assumidos na expectativa de que os benefícios futuros pudessem suplantá-los, o que se mostrou verdadeiro.

Para isso, o processo de desenvolvimento docente (segundo momento) criado e desenvolvido por profissionais sensíveis e sintonizadas com nossa realidade exerceu papel fundamental. A customização da capacitação, altamente apropriada à nossa necessidade, promoveu a valorização da atuação do mentor e da sua autoconfiança. Entendemos que a apropriação de mentores e alunos do processo e do projeto da mentoria foi marcante.

Prova dessa apropriação foi o produto gerado pelos partícipes ao trazerem sugestões qualificadas para a formatação das linhas norteadoras para o módulo Estudos Orientados, altamente adequadas para cada ano do curso. Além disso, aprovaram, em sua maioria, a continuidade da mentoria no internato (terceiro momento).

Obviamente, em se tratando de uma realidade tão complexa como o encontro entre mentor e mentorado, não há unanimidade quando a mentoria é avaliada. A aprovação ou não de quem a vivencia é também encontrada em outros estudos e deve estar sempre no horizonte de quem está atuando como mentor ou mentorado ${ }^{3-5}$.

Há diferentes desenhos e tipos de programa de mentoria descritos na literatura ${ }^{6-9}$. No entanto, nossa escolha por um programa oferecido com carga horária e ambiente protegidos, presenciais ou virtuais, em acompanhamento longitudinal, simultâneos para os três anos, com flexibilidade de conteúdos e de forma de atuação, com foco importante no profissionalismo, na autonomia e na remediação de desempenhos insatisfatórios vem provando ter sido acertada. Porém, somente o acompanhamento e a avaliação do processo em médio prazo nos darão subsídios para futuras mudanças, que certamente ocorrerão, já que lidamos com a realidade mutável em sua natureza.

A abertura para a escuta criteriosa dos envolvidos, especialmente com respeito às atividades de desenvolvimento docente para a mentoria, foi fundamental para a implantação e consolidação do programa ${ }^{10}$. Contamos hoje com 52 professores (24,3\% do total do corpo docente) capacitados a exercer a mentoria, 23 deles participando de mais de uma edição. É interessante ressaltar que alguns, ao participarem da capacitação, já se autoavaliaram como não tendo o perfil adequado para exercerem a mentoria, mais um ponto positivo para essa potente intervenção. Além disso, sem a capacitação competente e adequada ao desenho escolhido, e sem o espaço para trocas de experiências, não há avanços para que o programa se consolide e se transforme em uma cultura da mentoria, conforme preconizam Choi et al. ${ }^{11}$, desejável como um contraponto à realidade extremamente mutável e altamente competitiva com a qual nos deparamos. É o mundo VUCA -acrônimo de volatile (volátil), uncertain (incerto), complex (complexo) e ambiguous (ambíguo) - que exige novas competências diante dos desafios apresentados e a agilidade na troca de informações (falsas e verdadeiras), como no tempo atual ${ }^{11,12}$.

A mentoria não tem um conceito exato e fixo ${ }^{13}$. Podemos dizer que o nosso conceito de mentoria está se construindo e, conforme essa construção acontece, vemos de forma nítida a importância da rede de sustentação entre os mentores.

A curiosidade foi a principal responsável por conduzir alguns mentores à prática da mentoria, e vários se consideram gratificados nesse novo papel. Indo na direção contrária do dístico do brasão da cidade de São Paulo, onde se lê "Non ducor, duco" ("Não sou conduzido, conduzo"), criado pelo 
poeta Guilherme de Almeida em parceria com o pintor José Wasth Rodrigues, que venceram o concurso para estabelecer o brasão em 1917, a sensação é de que na mentoria o conduzido sou "eu, o mentor". E avançando no tempo, podemos nos apoiar em Paulo Freire" (p.25), quando afirma que "quem forma se forma e re-forma ao formar e quem é formado formase e forma ao ser formado".

\section{CONCLUSÕES}

Longevo, o curso de Medicina da PUC-SP tem sido constantemente revitalizado por reformas curriculares. Mais recentemente, a oportunidade aliada à necessidade, o desenvolvimento docente apropriado e os avanços embasados pela avaliação do processo formataram a criação e o desenvolvimento da prática da mentoria no curso. A escolha por um modelo alinhado com o PPC do curso e com características singulares vem se mostrando acertada pela consulta realizada com os envolvidos na atividade (alunos e professores), prevendo sua continuidade futura e lapidando seu desenvolvimento.

Nossa experiência permite inferir que, por suas características complexas e mutáveis, a mentoria deve ser construída em estreita relação com a comunidade e ajustada às necessidades de cada curso e do seu projeto pedagógico.

\section{CONTRIBUIÇÃO DOS AUTORES}

Todos os autores participaram da concepção do projeto, do seu desenvolvimento e da coleta de informações. A redação do relato de experiência foi feita por Maria Helena Senger e Fernando Antonio de Almeida, e todos os autores participaram da revisão e aprovação do texto final.

\section{FINANCIAMENTO}

Declaramos que não houve financiamento para a realização desta pesquisa.

\section{REFERÊNCIAS}

1. Pontifícia Universidade Católica de São Paulo. PPC. Sorocaba: PUC-SP; 2018.

2. Botti SHO, Rego S. Preceptor, supervisor, tutor e mentor: quais são seus papéis? Rev Bras Educ Med. 2008;32(3):363-73.

3. Gonçalves MCN, Bellodi PL. Ser mentor em medicina: uma visão arquetípica das motivações e transformações na jornada. Interface (Botucatu). 2012;16(41):501-14.

4. Martins AF, Bellodi PL. Mentoring in medical students: a humane and developmental experience. Interface (Botucatu). 2016;20(58):715-26.

5. Ziegler A, Gryc KL, Hopp MDS, Stoeger H. Spaces of possibilities: a theoretical analysis of mentoring form a regulatory perspective. Ann NY Acad Sci. 2021;1483(1):174-98.

6. Frei E, Stamm M, Buddeberg-Fischer B. Mentoring programs for medical students - a review of the PubMed literature 2000-2008. BMC Med Educ 2010;10:32-45. doi: 10.1186/1472-6920-10-32.

7. Nimmons D, Giny S, Rosenthal J. Medical student mentoring programs: current insights. Adv Med Educ Pract. 2019;10:113-23.

8. Farkas AH, Allenbaugh J, Bonifacino E, Turner R, Corbelli JA. Mentorship of US medical students: a systematic review. J Gen Intern Med. 34(11):2602 9. doi: 10.1007/s11606-019-05256-4.

9. Pinilla $S$, Nicolai L, Gradel M, Pander T, Martin D, Fischer R, et al. Undergraduate medical students using facebook as a peer-mentoring platform: a mixed-methods study. JMIR Med Educ. 2015;1(2):e12.

10. Ramani S, Gruppen L, Kachur EK. Twelve tips for developing effective mentors. Med Teach. 2006;28(5):404-8.

11. Choi AMK, Moon JE, Steinecke A, Prescott JE. Developing a culture of mentorship to strengthen Academic Medical Centers. Acad Med. 2019;94(5):630-3.

12. Maini $A$, Saravanan $Y$, Singh TA, Fyfe $M$. Coaching skills for medical education in a VUCA world. Med Teach. 2020;42(11):1308-9.

13. Mullen CA, Klimaitis CC. Defining mentoring: a literature review of issues, types, and applications. Ann NY Acad Sci. 2021;1483(1):19-35.

14. Freire P. Pedagogia da autonomia. Saberes necessário à prática educativa. 9a ed. São Paulo: Paz e Terra; 1998.

\section{CONFLITO DE INTERESSES}

Os autores declaram não haver conflito de interesses neste estudo. 